\title{
FUNÇÕES BASE ESPECIAIS PARA A SOLUÇÃO DE PROBLEMAS DE ESPALHAMENTO ELETROMAGNÉTICO POR CORPOS DE REVOLUÇÃO
}

\author{
Fernando Lisboa Teixeira ${ }^{1}$ e José Ricardo Bergmann ${ }^{2}$ \\ ${ }^{1}$ EMBRATEL - Departamento de Transmissão Satélite \\ ${ }^{2}$ CETUC/PUC-Rio - Centro de Estudos em Telecomunicações da \\ Pontifícia Universidade Católica do Rio de Janeiro
}

\begin{abstract}
Resumo: $O$ uso de funções base especiais é investigado na solução de equações integrais, utilizando-se o Método dos Momentos, para a análise do espalhamento eletromagnético por objetos condutores elétricos perfeitos com simetria de revolução. O desempenho em termos de acurácia relativa e requerimentos computacionais de duas classes de funções (B-splines e funções limitadas em banda) é avaliada contra aquele apresentado pelas funções base comumente utilizadas - funções trigonométricas globais e funções locais. Atenção particular é dada ao comportamento resultante da corrente induzida próxima às bordas. Questões relativas à localização espacial e espectral das funções base são consideradas. Exemplos incluem o espalhamento por antenas refletoras.
\end{abstract}

\begin{abstract}
The use of special base functions is investigated in the solution of integral equations by the Method of Moments for the analysis of electromagnetic scattering produced by perfectly conducting objects with circular symmetry. Performance with respect to relative precision and computational requirements for two classes of functions (B-spline and band-limited) is evaluated in comparison with that achieved by local and global trigonometric functions. In particular, behavior caused by near-edge induced currents is investigated. Also considered are issues related to spatial and spectral localization of base functions. Examples are given which include scattering by reflector antennas.
\end{abstract}

Palavras Chaves: Espalhamento Eletromagnético, Método dos Momentos, Equações Integrais, Antenas Refletoras, Antenas Axialmente Simétricas

\section{INTRODUÇÃO}

O tratamento numérico de problemas de espalhamento e/ou radiação de fronteira aberta no domínio da frequência é usualmente feito empregando-se uma formulação baseada em equações integrais. O Método dos Momentos (MM) é um procedimento geral de redução de uma equação integral (linear) para um sistema de equações lineares, através da projeção do problema original em um espaço de Hilbert para um espaço de dimensão finita $\mathbb{C}^{n}$ [1]. A matriz associada do sistema (matriz impedância) entretanto, é usualmente densa e de inversão computacionalmente intensiva. Para a redução do custo computacional (espaço de memória e tempo de CPU), um aspecto crucial do MM é a escolha adequada das funções base e testes adequadas. Duas classes principais de funções base comumente empregadas no MM podem ser identificadas: funções base de domínio local (suporte compacto) [2-4] e funções base globais [4-6]. Funções base de domínio global têm um caráter mais especializado, sendo utilizadas em problemas específicos para atingir maior eficiência computacional. Necessitam, no caso do espalhamento por objetos suaves, de uma taxa de amostragem $(\sigma)$ equivalendo a cerca de duas a três funções base por comprimento de onda. Funções base locais, por sua vez, são geometricamente mais flexíveiș, sendo mais práticas para a análise de geometrias mais gerais. Em compensação, elas necessitam de uma taxa de amostragem bem mais alta, de cerca de dez funções base por comprimento de onda $(\sigma \approx 10)$ como regra geral, o que torna proibitiva a análise de objetos espalhadores de grandes dimensões elétricas.

Para certos tipos de problemas de espalhamento por condutores elétricos perfeitos [4,5], a maior eficiência computacional obtida com o emprego de funções base globais está relacionada com as características espectrais da corrente elétrica induzida. De particular interesse, devido à sua importância prática, são os objetos espalhadores de geometria suave, i.e., cujo raio de curvatura local é maior que um comprimento de onda. Para tais objetos, uma hipótese razoável constitui-se assumir que as corrente induzidas possuem um espectro essencialmente limitado em frequência espacial $(|\vec{k}|$ número de onda), podendo ser aproximadas por funções de espectro confinado a um número de onda $\mathrm{k}_{\mathrm{m}}\left(|\vec{k}| \leq k_{m}\right)$ (especialmente no contexto do espalhamento em campo distante). Argumentos ainda heurísticos são destacados em [7]. O problema da localização espectral da radiação eletromagnética já foi extensivamente discutido em [8]. As funções base de domínio global usualmente empregadas (e. g., funções senoidais) possuem um espectro concentrado em baixas

\footnotetext{
${ }^{1}$ Endereço atual: Center for Computational Electromagnetics, University of Illinois at Urbana-Champaign

${ }^{2}$ Trabalho foi financiado no âmbito do contrato PUC-TELEBRÁS
} 
freqüências, tornando-se, portanto, candidatas naturais a uma expansão eficiente das correntes incógnitas (número reduzido de funções base por comprimento de onda).

Quando comparadas às funções base globais, a redução da região de suporte é uma das características atraentes das funções bases. Esta característica implica na diminuição do tempo de computação para o cálculo das integrais que compõem os elementos da matriz impedância, pois somente uma pequena região do objeto espalhador é envolvida por cada par função base/função teste (relacionado biunivocamente com determinado elemento da matriz). Além disto, devido ao fato de que o domínio das funções globais envolve todo o objeto espalhador, o tempo de CPU requerido para o cálculo de cada elemento é, neste caso, dependente das dimensões elétricas do objeto. Isto resulta no aumento da complexidade computacional do problema em função do tamanho elétrico/frequência de $O\left(D^{2}\right)$ para $O\left(D^{4}\right)$, onde $D$ é a dimensão característica do problema.

Destas observações pode-se traçar o interesse no desenvolvimento de um esquema numérico capaz de combinar os aspectos atrativos das funçōes locais e globais e evitar suas deficiências. Este esquema corresponderia ao uso de funções base de suporte espacial limitado e, simultaneamente, de espectro limitado. Idealmente, este objetivo não é realizável, visto que a transformada de Fourier de qualquer função de suporte compacto possui, necessariamente, suporte infinito. Dentro do contexto do espalhamento por condutores elétricos perfeitos com simetria de revolução, este trabalho apresenta a aplicação de duas estratégias que tem como objetivo otimizar o compromisso entre suporte e espectro.

A primeira estratégia consiste no emprego de funções base limitadas em banda $[7,9,10]$, que são basicamente funções do tipo sampling (de espectro por natureza limitado) adaptadas para possibilitar truncamento no domínio espacial sem que isto introduza componentes relevantes de alta frequência e sem que a degradação da ortogonalidade tenha consequiências práticas.

A segunda estratégia consiste no emprego de B-splines [11], que possuem localização quase ótima no espaço de fase $\vec{r} \times \vec{k}$ (com convergência assintótica para funções Gaussianas, otimamente localizadas) e simplicidade analítica que permite a extração exata das singularidades que surgem nas integrais envolvidas.

Para caracterizar a redução computacional obtida, é feita uma comparação do desempenho de cada opção quando aplicada a diferentes geometrias. Particular ênfase é dada ao caso de espalhamento por geometrias representativas de antenas refletoras axialmente simétricas (corpo de revolução aberto). Tais geometrias são extremamente populares devido à facilidade de fabricação e montagem, e por satisfazerem condições usualmente impostas sobre o diagrama de radiação (e. g., níveis de isolamento para polarização cruzada). Particularmente a primeira característica conduz ao emprego de superfícies axialmente simétricas não só em antenas de configurações axialmente simétricas [12], como também em configurações mais gerais, como em sistemas off-set [13].

\section{SOLUÇÃO DO ESPALHAMENTO POR CORPOS DE REVOLUÇÃO PELO MÉTODO DOS MOMENTOS}

Nesta seção a formulação da análise do espalhamento por objetos condutores perfeitos com simetria de revolução pelo MM no domínio da frequência é resumidamente discutida. Para um discussão mais detalhada, recomenda-se a consulta da referência [2].

Um corpo de revolução (CR) é gerado através da rotação do arco gerador $C$ em torno do eixo z. Na implementação numérica, o arco $C$ é aproximado por uma sequêencia de segmentos lineares $\varepsilon_{\mathrm{i}}$. Qualquer ponto sobre a superfície do CR pode ser descrito por duas coordenadas (ortogonais): 0 ângulo azimutal $\phi$ e o comprimento de arco $t$ ao longo da curva $C$ (normalizado com relação ao comprimento de onda do espaço livre). As Figura 1(a) e (b) ilustram a geometria típica de um refletor com simetria de revolução e o sistema de coordenadas utilizado para representação do $C R$.

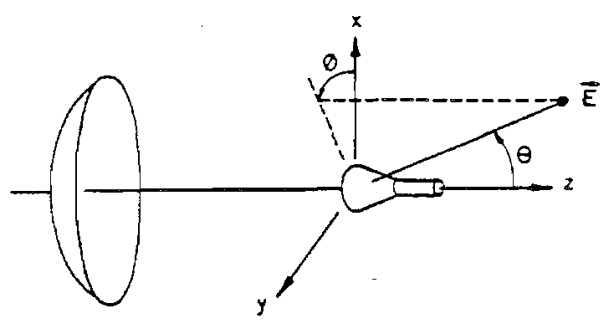

(a) Geometria do Refletor

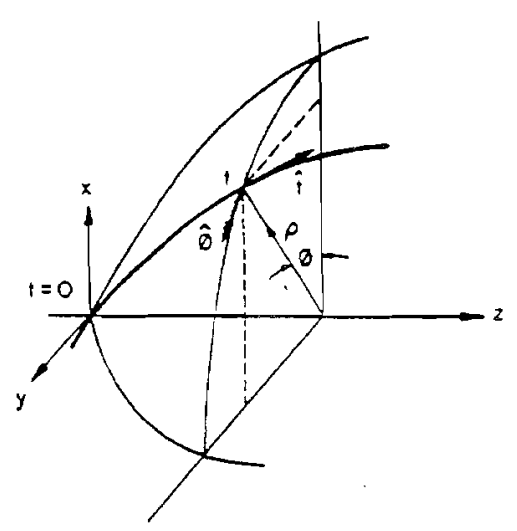

(b) Sistema de Coordenadas

Figura 1. (a) Geometria típica de refletor com simetria de revolução; (b) Parametrização em t e $\phi$ para corpo de revolução. 
Dado um campo elétrico incidente $\overrightarrow{\mathrm{E}}_{\text {inc }}$ sobre o $\mathrm{CR}$, a solução do problema (determinação da corrente elétrica induzida sobre a superfície) segue pela aplicação da pertinente condição de contorno do problema (campo elétrico tangencial total sobre a superfície condutora igual a zero) e pela expressão do campo elétrico espalhado em função da corrente induzida (incógnita). A equação resultante denomina-se Equação Integral para o Campo Elétrico (EICE) e relaciona a corrente induzida $\vec{J}$ ao campo elétrico incidente $\overrightarrow{\mathrm{E}}_{\text {inc }}$ através de uma equação integ̣al (vetorial) de Fredholm do primeiro tipo:

$\hat{n} \times \vec{E}_{i n c}=\hat{n} \times L(\vec{J})=\hat{n} \times j \omega \mu \iint_{s} \bar{J}\left(\bar{r}^{\prime}\right) G\left(\bar{r}, \bar{r}^{\prime}\right) d s^{\prime}+\frac{j}{\omega \varepsilon} \hat{n} \times \bar{\nabla}_{s} \iint_{s}\left(\vec{\nabla}_{s}^{\prime}, \vec{J}\right) G\left(\bar{r}, \bar{r}^{\prime}\right) d s^{\prime}$

onde $\bar{n}$ é o vetor unitário normal a superfície ,wé a frequêencia em radianos, $\mu$ é a permeabilidade do meio, $d s^{\prime}$ o elemento de área sobre $S, L(J)$ o operador integro difrencial e $G\left(\vec{r}, \vec{r}^{\prime}\right)$ é a função de Green do espaço livre:

$$
G\left(\vec{r}, \vec{r}^{\prime}\right)=\frac{e^{-j k o\left|\vec{r}-\vec{r}^{\prime}\right|}}{4 \pi\left|\vec{r}-\vec{r}^{\prime}\right|}
$$

A decomposição da corrente induzida nas suas componentes (ortogonais) ao longo de $\phi$ e $t$, resulta em um conjunto de duas equações integro-diferenciais acopladas descritas como:

$$
\left(L^{t t} \hat{t} \hat{t}+L^{t \phi} \hat{t} \hat{\phi}+L^{\phi \phi} \hat{\phi} \hat{t}+L^{\phi \phi} \hat{\phi} \hat{\phi}\right) \cdot\left(J_{t} \hat{t}+J_{\phi} \hat{\phi}\right)=E_{t}^{i n c} \hat{t}+E_{\phi}^{i n c} \hat{\phi}(3)
$$

onde $\hat{t}$ e $\hat{\phi}$ são vetores unitários na direção azimutal e tangencial e $L^{p q}$ são os operadores escalares integrodiferenciais $(\mathrm{p}, \mathrm{q}=\mathrm{t}, \phi)$. Para a aplicação do $\mathrm{MM}$ a este conjunto de equações expressa-se, primeiramente, a corrente incógnita em termos de um conjunto adequado de funções base $\vec{b}_{\mathrm{mi}}^{\mathrm{p}}(\mathrm{t}, \phi)$ previamente escolhidas:

$$
\begin{aligned}
& \vec{J}(t, \phi)=\sum_{m=-M}^{N}\left[\sum_{i=1}^{N t} I_{m i}^{t} \vec{b}_{m i}^{t}(t, \phi)+\sum_{i=1}^{N \phi} I_{m i}^{\phi} \vec{b}_{m i}^{\phi}(t, \phi)\right] \\
& \vec{b}_{m i}^{t}(t, \phi)=\frac{b_{i}^{t}(t)}{\rho(t)} e^{j m \phi} \hat{t}
\end{aligned}
$$

onde $I^{p}{ }_{m i}$ são os coeficientes a determinar. $O$ fator $1 / \rho(t)$ na componente $t$ cancela $O$ fator $\rho(t)$ que surge devido ao elemento de superfície $\mathrm{d} S=\rho(\mathrm{t})$ dodt. A equação integrodiferencial em (1) é transformada em uma equação matricial inserindo-se (4) em (3) e promovendo um produto interno das equações resultantes com um conjunto de funções (teste), complexas conjugadas às funções base (método de Galerkin). O produto interno é operacionalmente definido como sendo a integral sobre a superfície espalhadora do produto escalar de duas funções vetoriais. Com a escolha de dependência harmônica no ângulo de azimute e pela simetria rotacional do problema, ocorre um desacoplamento natural entre os diferentes modos (índices $m$ ) em (4). $\mathrm{O}$ sistema linear resultante exibe uma estrutura diagonal por blocos e cada modo pode ser calculado separadamente, reduzindo consideravelmente o esforço computacional para a solução completa do problema. O sistema linear para o mésimo modo é dado por:

$\left[\begin{array}{ll}\mathrm{Z}_{\mathrm{m}}^{\mathrm{tt}} & \mathrm{Z}_{\mathrm{m}}^{\mathrm{t} \phi} \\ \mathrm{Z}_{\mathrm{m}}^{\mathrm{\phi t}} & \mathrm{Z}_{\mathrm{m}}^{\phi \dot{\varphi}}\end{array}\right]\left[\begin{array}{c}\mathrm{I} \\ \mathrm{m} \\ \mathrm{I}_{\mathrm{m}}^{\phi}\end{array}\right]=\left[\begin{array}{c}\mathrm{V}_{\mathrm{m}}^{\mathrm{t}} \\ \mathrm{V}_{\mathrm{m}}^{\phi}\end{array}\right]$

$\left(Z_{m}^{p q}\right)_{i j}=\left\langle L\left(\vec{b}_{m j}^{q}\right), \vec{b}_{m i}^{p^{*}}\right\rangle$

$\left(\mathrm{V}_{\mathrm{m}}^{\mathrm{p}}\right)_{\mathrm{i}}=\left\langle\overrightarrow{\mathrm{E}}^{\mathrm{inc}}, \overrightarrow{\mathrm{b}}_{\mathrm{mi}}^{\mathrm{p}^{*}}\right\rangle$

onde <,> denota produto interno. As submatrizes $\mathrm{Z}_{\mathrm{m}}^{\mathrm{pq}}$ compõem a matriz impedância do problema. O vetor no lado direito de (5) é o vetor excitação. Expressões explícitas para os elementos da matriz impedância e para os vetores excitação são encontradas em [25]. A solução para a equaçăo (5) determina a corrente induzida dada por (4).

O número de modos necessários para expressar adequadamente um problema específico pode ser determinado através de um estudo de convergência. Pode-se mostrar [14] que, no caso de ondas planas com direção de incidência formando um ângulo $\theta$ com o eixo de simetria do $\mathrm{CR}$, o número mínimo necessário de modos será dado por $\mathrm{M}=2 \pi\left(\rho_{\mathrm{m}} / \lambda\right) \operatorname{sen} \theta_{\mathrm{t}}$, onde $\lambda$ é o comprimento de onda e $\rho_{\mathrm{m}}$ é a máxima extensão radial do $C R$. No caso de onda plana com incidência axial, apenas os modos $m= \pm 1$ serão excitados.

\section{FUNÇÕES BASE CONVENCIONAIS}

As funções base $b_{i}^{t}(t)$ e $b_{i}^{\phi}(t)$ comumente utilizadas neste tipo de problema incluem funções base locais e funções base globais.

Funções base locais empregadas consistem essencialmente de dois tipos distintos. O primeiro consiste de pulsos deslocados (staggered pulses) [3], cuja característica fundamental é a grande flexibilidade geométrica que proporciona em problemas mais gerais. $\mathrm{O}$ segundo consiste de pulsos na componente $\phi$ e triângulos na componente t [2]. Este é o conjunto mais simples para evitar aproximações (diferenças finitas) nos operadores diferenciais em (1). Em termos de eficiência computacional ambos os tipos apresentam essencialmente o mesmo desempenho. Neste trabalho, para fins de comparação será adotado o segundo tipo, definido em [2].

Funções base globais, aqui adotadas, são funções senoidais definidas sobre toda a curva $C$ como em [5], consistindo de senos para a componente $\phi$ e cossenos para a componente $t$. 


\section{FUNÇÕES BASE LIMITADAS EM BANDA}

Nesta seção discute-se o emprego de funções base limitadas em banda para a dependência na coordenada t. Para CR, utiliza-se uma versão adaptada das funções introduzidas em [7] no contexto de expalhamento bidimensional, sendo escritas como:

$$
\begin{aligned}
b_{i}^{t}(t) & =\frac{t}{\rho(t)} \operatorname{sinc}\left[\alpha R_{N}\left((t / T)-\tau_{i}\right)\right] \operatorname{sinc}\left[R_{N}\left((t / T)-\tau_{i}\right)\right] \\
; i & =1, \ldots, N_{t}
\end{aligned}
$$

$$
b_{i}^{\phi}(t)=\operatorname{sinc}\left[\alpha R_{N}\left((t / T)-\tau_{i}\right)\right] \operatorname{sinc}\left[R_{N}\left((t / T)-\tau_{i}\right)\right]
$$$$
=1, \ldots, N_{0}
$$

onde $\operatorname{sinc}(x)=\operatorname{sen}(\pi x) / \pi x$.

A Figura 2 ilustra este tipo de função base.. $O$ fator $R_{N}=$ $N_{t}$ está associado à taxa de amostragem, dada por $\sigma=N_{t} / T$ e a localização das funções base é definida por $\tau_{\mathrm{i}}=(\mathrm{i}-$ 1) $/ R_{N}$. T é o comprimento total do arco gerador $C$. O conjunto acima forma uma expansão exata de qualquer função de espectro limitado $\mathrm{a}(1-\alpha) \sigma / 2,0<\alpha<1$ e dessa forma está amostrada a uma taxa $1 /(1-\alpha)$ maior que a taxa de Nyquist. $O$ fator $\alpha$ controla o compromisso entre $o$ grau de superposição entre as funçōes base (que afeta o tempo necessário para calcular a matriz impedância) e a taxa de amostragem requerida (relacionada com a memória necessária para armazenar a matriz impedância). A escolha $\alpha=0.3$ é utilizada em todo este trabalho [7].

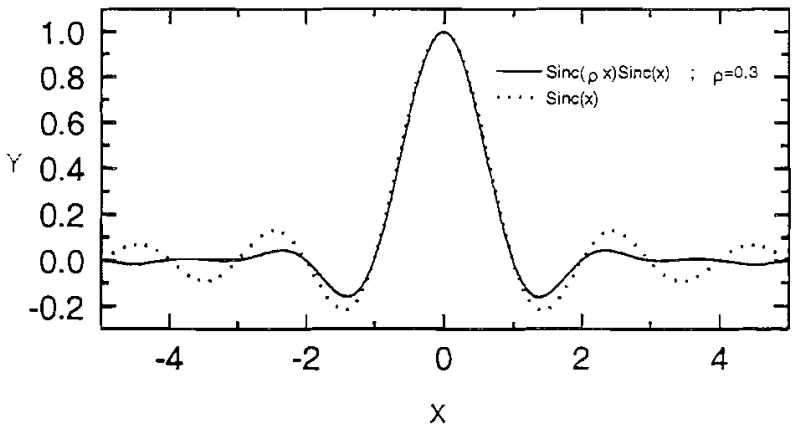

Figura 2. Funções limitadas em banda

O decaimento quadrático que estas funções apresentam possibilita um truncamento no primeiro zero do fator $\operatorname{sinc}\left[a R_{N}\left((t / T)-\tau_{i}\right)\right]$ sem que se introduza componentes relevantes de alta frequência. Este truncamento, por sua vez, permite que o cálculo das integrais que definem os elementos da matriz impedância seja feito sobre um região limitada do objeto, o que torna o tempo de cálculo de cada elemento independente do tamanho elétrico do objeto, da mesma forma que no caso de função base local. A escolha de $R_{N}=N_{t}$ (um inteiro) implica a priori uma componente longitudinal da corrente elétrica nula na borda da superfície (como é exigido pelas condições de contorno). Esta expansão é suficientemente geral para tratar uma variedade muito grande de superfícies abertas (refletores). Um grau adicional de flexibilidade pode ser obtido ao variar-se localmente a taxa de amostragem $\sigma$, permitindo-se assim, uma amostragem mais crítica em regiôes do corpo espalhador onde se espera componentes de mais alta frequência (regiões de grande curvatura ou descontinuidades geométricas como bordas).

\section{B-SPLINES COMO FUNÇÕES BASE}

A localização em frequêencia espacial envolve um compromisso com a localização espacial da função base (pelas propriedades da transformada de Fourier). O emprego de funções base globais ou funçōes base limitadas em banda acarreta uma localização reduzida com um maior grau de superposição. Este fato inevitavelmente leva a um compromisso entre os requerimentos em termos de espaço de memória (localização na frequência espacial) e tempo de CPU para o cálculo da matriz impedância (localização espacial). Uma alternativa que surge naturalmente destas observações é a utilização de funçōes base que tenham localização ótima no espaço de fase $(\overrightarrow{\mathbf{r}}, \overrightarrow{\mathbf{k}})$.

A primeira vista, funções gaussianas seriam uma escolha natural, visto que apresentam exatamente estas características através de um produto de variâncias no espaço $(\overline{\mathbf{r}}, \overline{\mathbf{k}})$ mínimo. Entretanto, a aplicação deste tipo de função resulta na necessidade de truncamento em algum ponto. Este procedimento pode ser evitado observando-se que funções gaussianas podem ser bem aproximadas por Bsplines, que possuem suporte compacto. B-splines convergem assintoticamente para gaussianas quando a ordem da spline $n \rightarrow \infty$. Em verdade, B-splines cúbicas ( $n$

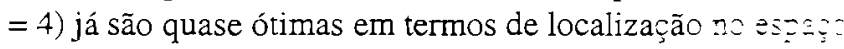
de fase, possuindo um produto de variâncias apenas $2 \%$ superior a aquele especificado pelo princípio da incerteza [15] e dentro de um erro de aproximação não superior a $3 \%$ em relação à função gaussiana, conforme ilustrado na Figura 3. Uma vantagem adicional das B-splines está relacionada com a extração pseudo-analítica das singularidades que surgem no cálculo das integrais na matriz impedância. Esta extração é realizada com o emprego de uma aproximação polinomial local para as funçōes base (cf. apêndice C). Esta aproximação pode ser feita exata, no caso de B-splines, pelo fato de que estas funções são localmente expressas como polinômios.

Para a construção de $B$-splines cúbicas $(n=4)$ em um domínio de interesse [a,b], o primeiro passo é definir uma partição de $\mathrm{K}+1$ pontos nodais (knots): $\left\{\mathrm{t}_{\mathrm{i}}\right\}_{\mathrm{i}=0, \mathrm{~K}}$, sendo $\mathrm{a}=$ $\mathrm{t}_{0}<\ldots<\mathrm{t}_{\mathrm{K}}=\mathrm{b}$. Pontos nodais adicionais são colocados nos pontos extremos do intervalo ( $k$ nots múltiplos): $\mathrm{t}_{-3}=\mathrm{t}_{-2}=\mathrm{t}$. $1=\mathrm{a}$ and $\mathrm{t}_{\mathrm{K}+1}=\mathrm{t}_{\mathrm{K}+2}=\mathrm{t}_{\mathrm{K}+3}=\mathrm{b}$.

Definindo 


$$
\gamma_{\mathrm{n}}(\mathrm{s} ; \mathrm{t})=(\mathrm{s}-\mathrm{t})_{+}^{\mathrm{n}-1} \equiv\left\{\begin{array}{cc}
(\mathrm{s}-\mathrm{t})^{\mathrm{n}-1} & \mathrm{~s} \geq \mathrm{t} \\
0 & \mathrm{~s}<\mathrm{t}
\end{array}\right.
$$

A B-spline (normalizada) de ordem n é dada pela n-ésima diferença dividida de $\gamma_{n}(s ; t)$ para $s$ em $t_{i}, \ldots, t_{i+n}$ para um $t$ fixo [16], i. e.,

$\beta_{n, i}(t)=\left(t_{i+n}-t_{i}\right) \gamma_{n}\left(t_{i}, \ldots, t_{i+n} ; t\right)$ para todo $i$

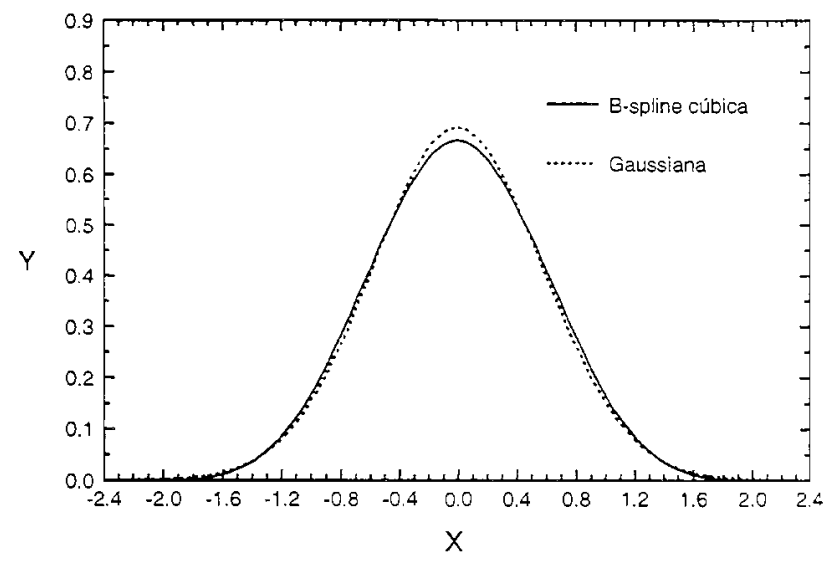

Figura 3. B-spline cúbica e a aproximação Gaussiana correspondente: $\psi(\mathrm{x})=(1.5 \pi)^{1 / 2} \exp \left(-1.5 \mathrm{x}^{2}\right)$.

A Figura 4 apresenta B-splines cúbicas (ordem $n=4$ ) no intervalo unitário ([a,b] $=[0,1])$ com espaçamento uniforme (que será o caso de todos os exemplos aqui tratados) e $\mathrm{K}=$ 8. Expressões explícitas para (10), podem ser encontradas em [17]. Cabe observar que a transfomada de Fourier da Bspline central de ordem n é dada por $\beta_{n}(t) \leftrightarrow \operatorname{sinc}^{n}(f)$, corroborando a localização espectral no domínio de freqüência espacial.

Uma terceira característica atrativa destas funções é o baixo grau de superposição em qualquer ponto da superfície (exceto próximo às bordas com múltiplos knots), onde no máximo três B-splines são não nulas. Próximo à borda, com - emprego de knots múltiplos, as funções tendem a aumentar sua localização e a possuir componentes de mais alta frequência. Isto é exatamente o que é necessário para uma melhor descrição da corrente induzida neste ponto (onde se espera comportamento singular [23]).

A expressão para as funções base em (4) aqui utilizadas em termos das B-splines cúbicas é escrita como:

$$
\begin{aligned}
& b_{i}^{t}(t)=t \beta_{4, i-1}(t) \quad i=1, \ldots, K-1=N_{t} \\
& b_{i}^{\phi}(t)=\beta_{4, i-1}(t) \quad i=1, \ldots, K=N_{\phi}
\end{aligned}
$$

cuja partição uniforme correspondente é dada por (onde $\left.\delta_{\mathrm{T}}=\mathrm{T} /(\mathrm{K}-2)\right)$ :

$$
\mathrm{t}_{\mathrm{i}}=\left\{\begin{array}{l}
-2 \delta_{\mathrm{T}} ; \mathrm{i}=-3, \ldots,-1 \\
(\mathrm{i}-2) \delta_{\mathrm{T}} ; \mathrm{i}=0, \ldots, \mathrm{K} \\
\mathrm{T} ; \mathrm{i}=\mathrm{K}+1, \ldots, \mathrm{K}+3
\end{array}\right.
$$

A partição acima implica que o máximo da primeira função base $\mathbf{b}_{i}^{t}(t), i=1$ coincide com o ponto $t=0$. As três primeiras funções ilustradas na Figura 4 (indicadas por $\mathrm{i}=$ 3,-2,-1 na figura) não estão incluídas na expansão (11) acima. $O$ fator $t$ em (11a) impõe a condição $J_{t}(t=0)=0 a$ priori (como no caso das funções limitadas em banda anteriormente definidas) no caso de uma borda neste ponto

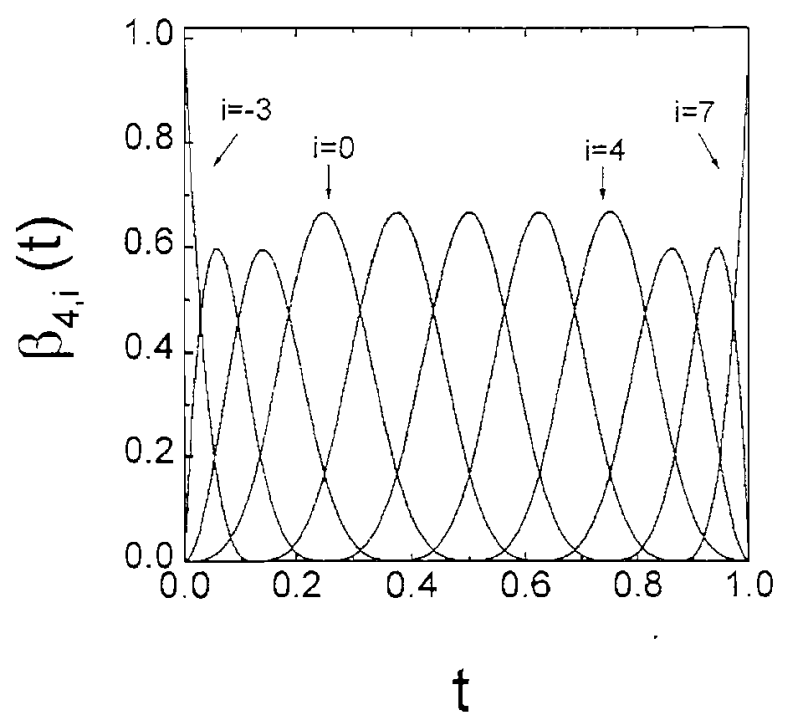

Figura 4. B-splines cúbicas por definidas sobre o intervalo $[0,1], K=8$ e espaçamento uniforme.

$(\rho(t=0) \neq 0)$ e cancela o fator $1 / \rho(t)$ no caso de $\rho(t=0)=0$. Também evita a instabilidade devido ao acoplamento da componente $\phi$ da corrente em regiōes onde a componente $\mathrm{J}_{\mathrm{t}}$ domina ambas as equações de (3) [18], como será ilustrado na próxima seção.

\section{RESULTADOS E APLICAÇÃO À ANÁLISE DE ANTENAS REFLETORAS}

A análise do espalhamento por antenas refletoras é usualmente feita através de aproximaçōes que não satisfazem às equações de Maxwell, como a ótica física (PO), a ótica geométrica (GO) e métodos assintóticos (GTD, PTD e correlatos). Tais aproximações são satisfatórias em problemas de síntese de refletores $[19,20]$ e para a maioria das aplicações de análise, principalmente quando a região de interesse do campo espalhado envolve apenas o lóbulo principal e os primeiros lóbulos laterais [21, 22]. Entretanto, para melhor precisar o nível de lóbulos mais afastados e/ou os efeitos da presença de estais e/ou de interação mútua (como, por exemplo, no caso de duplo refletores ou da interação refletor/alimentador em refletores compactos) a formulação do problema por equações integrais torna-se necessária. 
O primeiro exemplo a ser analisado nesta seção corresponde ao caso mais simples de CR aberto: um disco condutor elétrico perfeito infinitamente delgado e com $4 \lambda$ de diâmetro, sobre o qual incide (axialmente) uma onda plana de polarização linear. Assumindo, sem perda de generalidade, polarização na direção $x$, a componente $t$ é calculada no corte $\phi=0^{\circ}$ e a componente 0 no corte $\phi=90^{\circ}$. As Figs. 5(a) e (b) ilustram as correntes induzidas (componentes t e $\phi$ respectivamente), calculadas com função base globais, limitadas em banda e B-splines para uma mesma taxa de amostragem $\sigma=9.25$, correspondendo a $\mathrm{N}_{t} / \mathrm{N}_{\phi}=18 / 19$. O tempo de CPU necessário (normalizado) para cálculo da matriz impedância em cada caso encontra-se na Tabela 1. AFißüa 6 ilustra esquematicamente a distribuição de cortentes sobre o disco. Da Figura 5 , observa-se a claramente a tendência de comportamento prevista para a corrente na borda [23,24]: componente $\phi$ singular (e sobre a maior parte do objeto corretamente aproximada pela corrente da PO) e componente $t$ nula (e sobre a maior parte do objeto oscilando em torno do valor estimado pela corrente da PO). A Figura 5(c) mostra o resultado da análise (componente $\phi$ ) com as mesmas funções base a uma taxa de amostragem menor, $\sigma=5.25\left(\mathrm{~N}_{t} / \mathrm{N}_{0}=\right.$ 10/11). Observa-se a excelente concordância com a análise anterior, em especial se isolado o comportamento singular próximo à borda. A mesma análise para a componente t com esta taxa de amostragem apresenta um resultado relativamente ainda mais próximo ao da Figura 5(b) devido a ausência de comportamento singular. À exceção do comportamento próximo à borda da componente portanto, o espectro das correntes é essencialmente localizado em baixas freqüências $\left(|\vec{k}| \leq \mathrm{k}_{0}\right)$ o que destarte torna as representações que fazem uso deste fato mais eficientes em termos da taxa de amostragem necessária. Em linhas gerais, este mesmo tipo de comportamento estará presente nos refletores analisados a seguir.

\begin{tabular}{|c|c|}
\hline $\begin{array}{c}\text { Função Base }\left(\mathbf{N}_{\boldsymbol{t}} / \mathbf{N}_{\phi}=\right. \\
\mathbf{1 8} / \mathbf{1 9})\end{array}$ & $\begin{array}{c}\text { Tempo de CPU } \\
\text { normalizado }\end{array}$ \\
\hline Global & 100.0 \\
\hline Limitada em banda & 10.9 \\
\hline B-spline cúbica & 6.3 \\
\hline
\end{tabular}

Tabela 1. Tempo de CPU para cálculo da matriz impedância na análise do disco condutor por funções base globais, limitadas em banda e B-splines com $N_{t} / N_{\phi}=18 / 19$. Como o número de funções base é igual, os requerimentos de memória são idênticos.

O segundo exemplo consiste de um parabolóide (superfície mais comum em antenas refletoras simples) com diâmetro $D=10 \lambda$ e distância focal $f=4 \lambda$. A excitação corresponde a uma onda esférica incidente que simula $o$ diagrama de um alimentador corrugado com distribuição de potência do tipo $\cos ^{\mathrm{n}} \theta, n=2$, cujo centro de fase coincide com o foco do parabolóide. A distribuiçāo de corrente (componentes $t$ e 0 ) resultante se encontra nas Figuras 7 (a) e (b) calculada para diversos tipos de funções base e diferentes taxas de amostragem, juntamente com um resultado de referência obtido através da análise com um

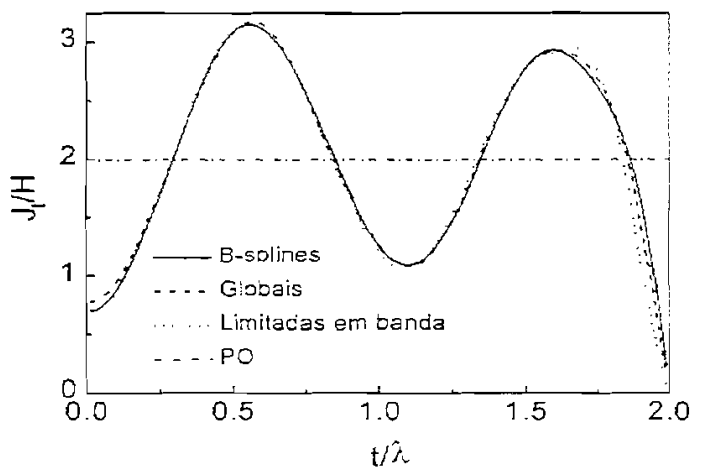

(a)

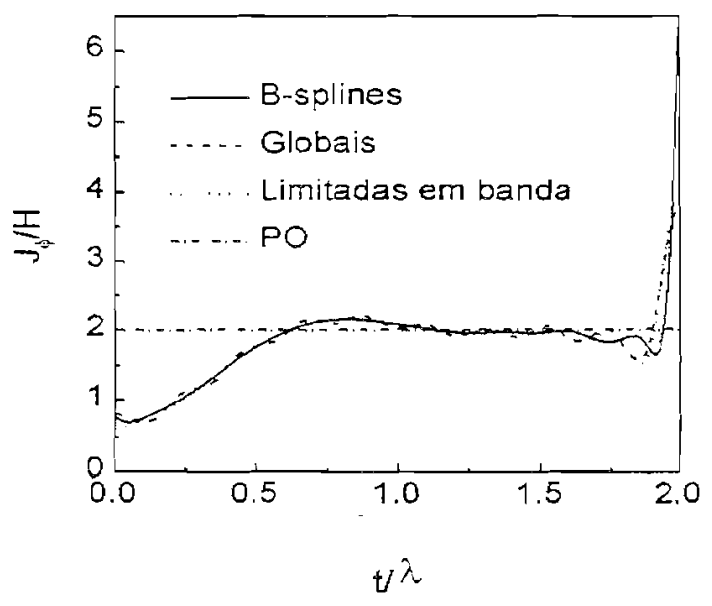

(b)

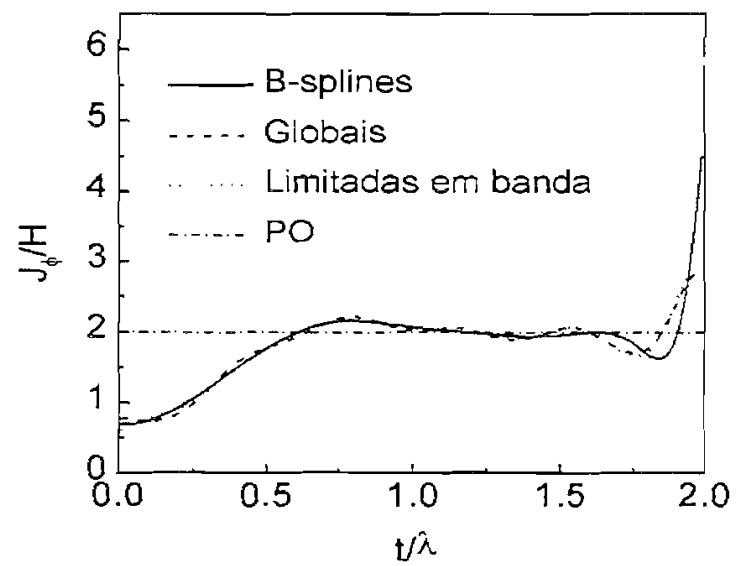

(c)

Figura 5. (a) Componente $t$ da densidade de corrente induzida sobre disco condutor com $D=4 \lambda$ para diversas funções base e $\sigma=9.25\left(\mathrm{~N}_{\mathrm{L}} / \mathrm{N}_{\phi}=18 / 19\right)$; (b) Componente $\dot{\phi}$ da densidade de corrente induzida sobre o disco condutos com $\mathrm{D}=4 \lambda$ para diversas func 2 base e $\sigma=9.25\left(\mathrm{~N}_{t} / \mathrm{N}_{0}=\right.$ 18/19). 


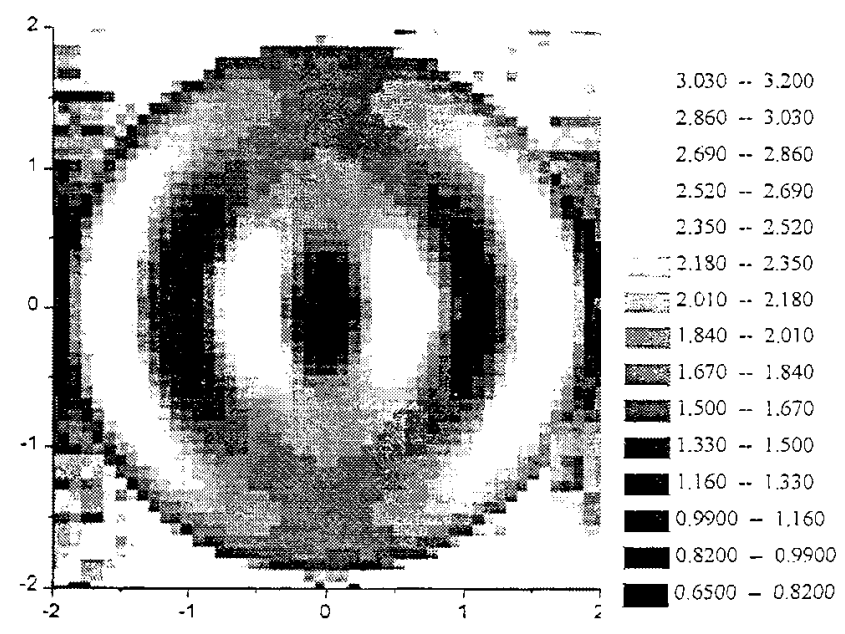

Figura 6. Ilustração esquemática da distribuição de densidade de corrente induzida sobre disco condutor $D=4 \lambda$

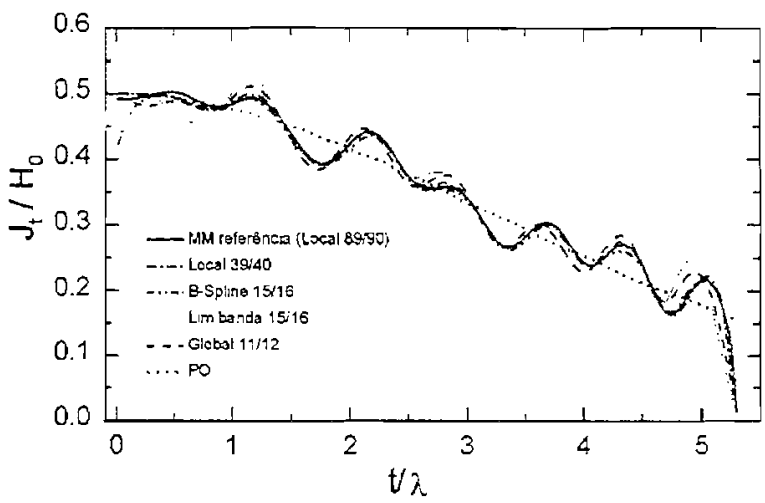

(a)

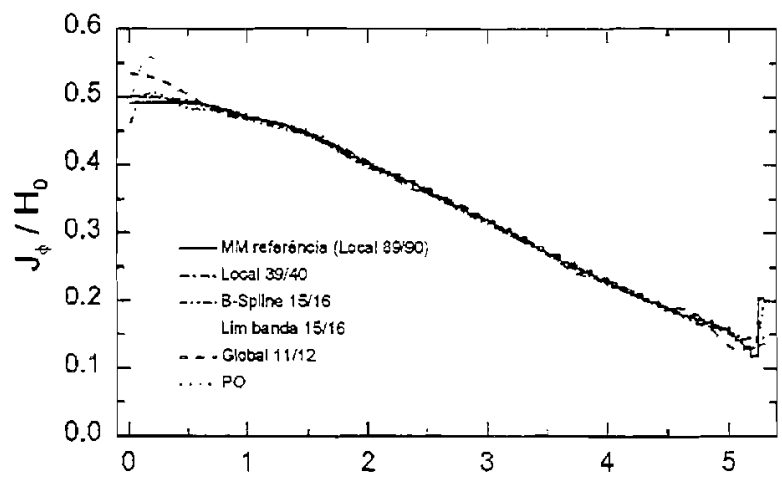

$t / \lambda$

(b)

Figura 7. (a) Componente $t$ da densidade de corrente induzida sobre refletor paraboloidal com $D=10 \lambda$ para diversas funções base e taxas de amostragem. (b) Componente $\phi$ da densidade de corrente induzida sobre refletor paraboloidal com $\mathrm{D}=10 \lambda$ para diversas funções base e taxas de amostragem.

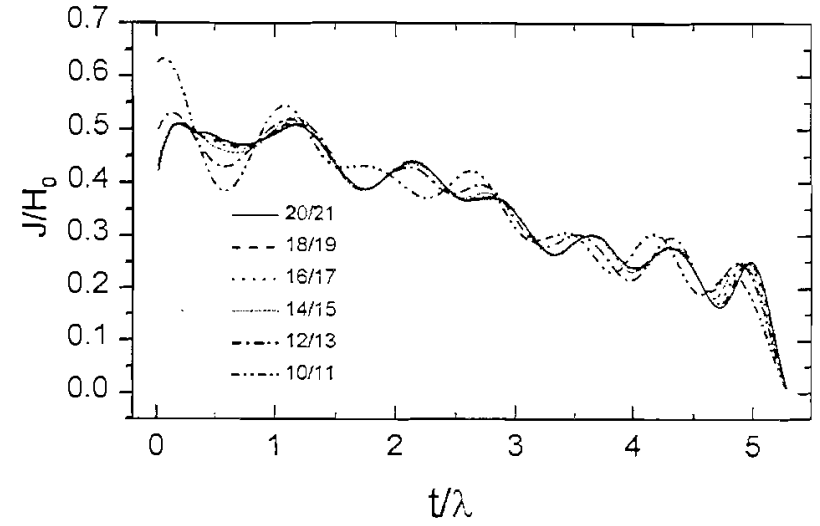

Figura 8. Convergência da componente longitudinal corrente utilizado ao empregar-se funções base limitz banda desde $\sigma=1.9$ até $\sigma=3.8$.

MM de alta densidade $(\sigma \approx 20$ funções base loz: : : :comprimento de onda). Pode-se observar que as $005:-2 . ;$. induzidas apresentam as características básicas observadas no caso do espalhamento pelo disco. $\therefore$. 8 ilustra o processo de convergência da compore:-: longitudinal da corrente ao variar-se a taxa de amostrageris no caso das funções limitadas em banda desde $\sigma \approx 1.9$ $\left(\mathrm{N}_{\mathrm{t}} / \mathrm{N}_{0}=10 / 11\right)$ até uma taxa duas vezes superior $\sigma=3.8$ $\left(N_{t} / N_{0}=20 / 21\right)$.

Pela estacionariedade da integral de radiação, variações e/ou discrepâncias de primeira ordem na corrente induzida terão um efeito apenas de segunda ordem sobre o campo espalhado $e$, consequentemente, sobre o radiado. Isto é ilustrado através do diagrama obtido para o parabolóide na Figura 9, onde a concordância entre o campo radiado (plano E) calculado utilizando-se um MM de alta densidade ( $\sigma=20$ funções base locais por comprimento de onda) e um MM com funções base limitadas em banda ou B-splines com taxa de amostragem tão baixa quanto $\sigma \approx 2.7$ (NFT/NFPHI $=15 / 16)$ apresentam excelente concordância. O diagrama previsto pela $\mathrm{PO}$ embora bastante preciso para os primeiros lóbulos secundários, já apresenta maiores discrepâncias para lóbulos mais afastados, em especial ao redor de $\theta=90^{\circ}$. O resultado para o plano $H$ apresenta uma concordância ainda maior entre os diversos esquemas numéricos, não sendo aqui apresentado.

A terceira configuração consiste de outro exemplo representativo de geometria empregada em antenas refletoras: uma superfície hiperboloidal com $D=10 \lambda$, com excentricidade $e=2$. e distância interfocal $2 c=18.334 \lambda$. O hiperbolóide é comumente empregado, por exemplo, como sub-refletor em sistemas de duplo-refletores Cassegrain, configuração largamente utilizada em antenas de estações terrenas para comunicações via-satélite. O campo incidente é o mesmo do caso anterior. A distribuição de corrente resultante sobre o hiperbolóide (componentes t e $\phi$ ) se encontra nas Figs.10(a) e (b). O campo espalhado no plano $E$ (campo primário não incluído) é representado na Figura 11. As mesmas observações efetuadas para o caso do 
refletor paraboloidal aqui se aplicam, sendo que a iluminação mais intensa da borda no caso do hiperbolóide provoca uma variação mais abrupta da componente $t$ da corrente do que no caso anterior. Este fato (devido as componentes de mais alta frequência aî geradas) responde pela discrepância ligeiramente maior observada entre as diversas representações. A Tabela 2 apresenta os requerimentos computacionais na análise dos refletores.

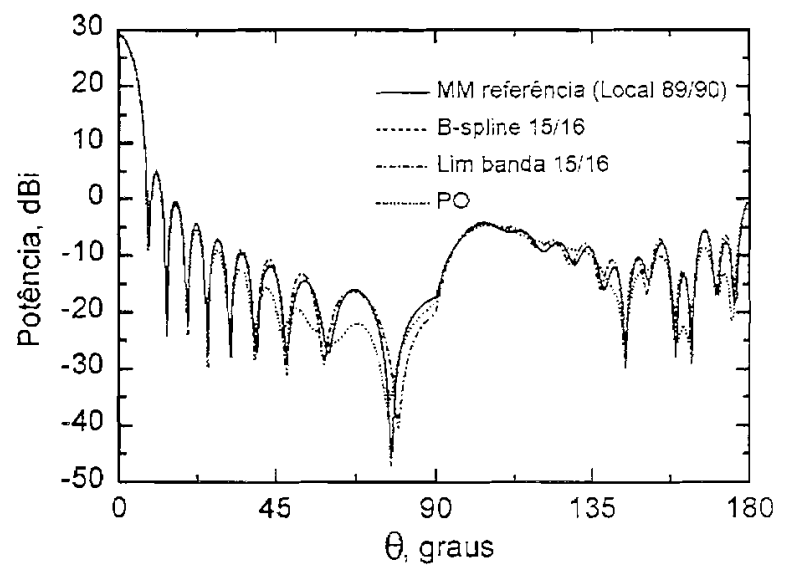

Figura 9. Diagrama do campo radiado (primário + espalhado) pelo refletor paraboloidal no plano $\mathrm{E}$.

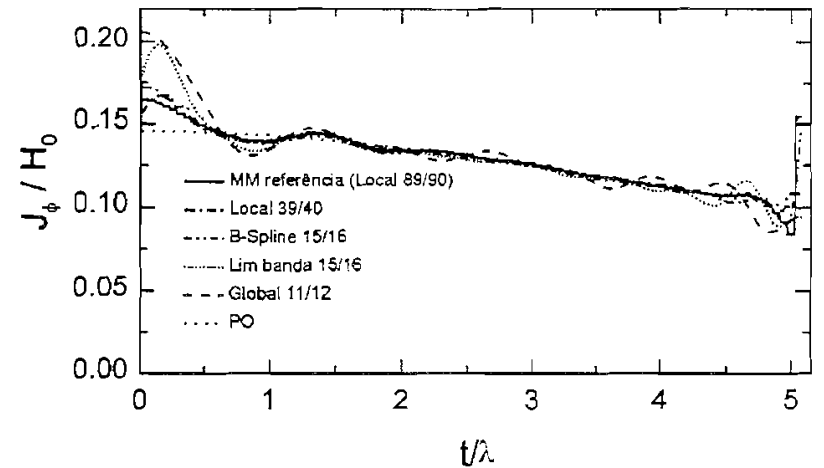

(a)

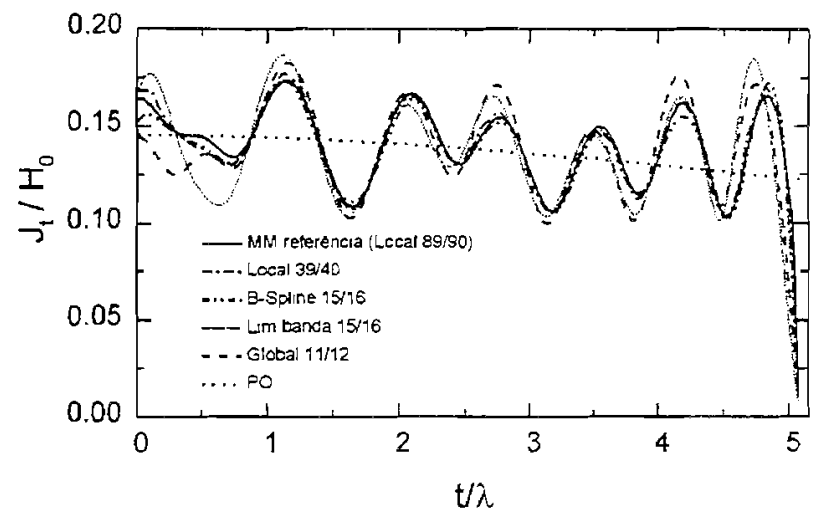

(b)

Figura 10. (a) Componente $t$ da densidade de corrente induzida sobre refletor hiperboloidal com $\mathrm{D}=10 \lambda$ para diversas funções base $e$ taxas de amostragem. (b) Componente $\phi$ da densidade de corrente induzida sobre refletor hiperboloidal com $D=10 \lambda$ para diversas funções base e taxas de amostragem.

Das Figs. 9 e 11, observa-se uma acurácia ligeiramente superior no cálculo do campo espalhado através da representação por B-splines do que aquele por funções limitadas em banda. Isto pode ser explicado pelo comportamento da corrente observado nas bordas, onde as B-splines reproduzem melhor (através do emprego de knots múltiplos) o comportamento singular da componente ô (que é aquela que, em última análise, influencia o resultado do

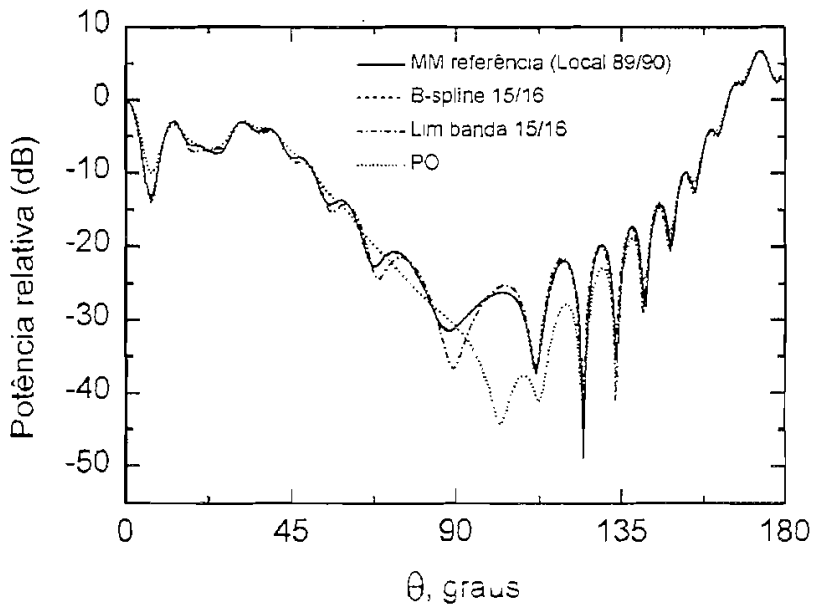

Figura 11. Campo espalhado (campo primário não incluído) pelo refletor hiperboloidal no plano $\mathrm{E}$.

campo no plano E). Novamente, os resultados para o plano $H$ não são aqui apresentados pois mostram os campos gerados pelas diversas representações com maior concordância. Este fato pode ser justificado observando-se que a componente $\mathrm{t}$ (que influencia o resultado no plano $\mathrm{H}$ ) não apresenta comportamento singular na borda, tendo, por conseguinte, espectro mais localizado do que a componente $\phi$.

\begin{tabular}{|c|c|c|c|}
\hline $\begin{array}{c}\text { Função } \\
\text { base }\end{array}$ & $\mathbf{N}_{t} / \mathbf{N}_{\phi}$ & $\begin{array}{c}\text { Espaço de } \\
\text { memória } \\
\text { ocupado pela } \\
\text { matriz } \\
\text { impedância(*) } \\
\text { - kbytes }\end{array}$ & $\begin{array}{c}\text { Tempo de } \\
\text { CPU para } \\
\text { cálculo da } \\
\text { matriz } \\
\text { impedância } \\
\text { (normalizado) }\end{array}$ \\
\hline Local & $89 / 90$ & 500.64 & 0.056 \\
\hline Local & $39 / 40$ & 97.52 & 0.033 \\
\hline Global & $11 / 12$ & 8.27 & 100.0 \\
\hline $\begin{array}{c}\text { Limitada } \\
\text { em banda }\end{array}$ & $15 / 16$ & 15.02 & 0.833 \\
\hline $\begin{array}{c}\text { B-spline } \\
\text { cúbica }\end{array}$ & $15 / 16$ & 15.02 & 0.473 \\
\hline
\end{tabular}

Tabela 2. Requerimentos computacionais para a análise dos refletores paraboloidal e hiperboloidal com diferentes funções base e taxas de amostragem 
Com respeito ao espaço de memória ocupado, diretamente proporcional ao número de funções base necessário, observa-se que, para níveis de convergência similares, funções base globais são mais econômicas. $O$ emprego de apenas $11 / 12$ funções base foi capaz de gerar resultados similares àqueles apresentados com 39/40 funções base locais. Entretanto, como já observado na discussão introdutória, o tempo de CPU necessário para o cálculo da matriz impedância é dramaticamente superior ao empregar-se funções base globais. As Tabelas 1 e 2 ilustram este fato, bem como o compromisso (em termos de tempo de CPU vs. espaço de memória requerido) obtido ao se utilizar as diversas funções base.

$O$ último exemplo considerado envolve um cilindro circular aberto com $1 \lambda$ de diâmetro e raio $a=1 / k_{0}$ sobre o qual incide uma onda plana linearmente polarizada com direção de propagação ao longo do eixo de simetria. Neste exemplo, o forte acoplamento existente entre as duas componentes da equação (3) e o comportamento dominante da componente $t$ pode acarretar oscilações espúrias (não físicas) na componente $\phi$ calculada numericamente, conforme já observado em [18]. Isto ocorre, por exemplo ao empregar-se B-splines sem o fator $t$ na expressão (11a) (e, em geral qualquer expansão local). Em contraste, o emprego da expansão (11) minimiza o comportamento dominante da componente $t$, estabilizando a componente $\rho$, conforme ilustrado na Figura 12.

\section{CONCLUSÕES}

A escolha adequada do par de funções base e teste é de grande importância na convergência e eficiência computacional do MM na solução de problemas de espalhamento eletromagnético. Apesar da pletora de escolhas possíveis, dois requerimentos básicos devem ser satisfeitos por uma escolha eficiente, quais sejam, possuir característica espectral (na frequência espacial) similar àquela apresentada pela corrente induzida (gerando assim uma solução convergente com poucos termos) e ser capaz de prover um tempo de CPU reduzido para o cálculo das integrais envolvidas.

Neste trabalho, diferentes escolhas de funções base foram investigadas no contexto do espalhamento por condutores elétricos perfeitos com simetria de revolução. Conceitos como localização espacial e espectral foram considerados. Mostrou-se que funções base de espectro limitado podem gerar representações bastante eficientes no

caso de objetos suaves embora o comportamento singular próximo a bordas seja em certa medida negligenciado. Neste aspecto, o emprego de B-splines com knots múltiplos provou ser mais adequado para uma representação mais correta das correntes do que funçōes base globais ou limitadas em banda.

A comparação do tempo de cálculo da matriz impedância evidenciou uma limitação básica das funções globais. Mesmo para refletores com diâmetro da ordem de $10 \lambda$, o tempo requerido por estas funções foi significativamente superior. Para refletores maiores, esta limitação será ainda mais evidente, pela dependência deste tempo com o tamanho característico $D$ do objeto ser mais crítica no caso das funções globais $\left(\mathrm{D}^{4}\right)$ do que nas outras opções $\left(\mathrm{D}^{2}\right)$

A possibilidade de eliminar o comportamento espúrio da corrente devido ao forte acoplamento nas componentes em (3) da equação integral do problema (1) também foi investigada. Em particular, o emprego de um fator linear

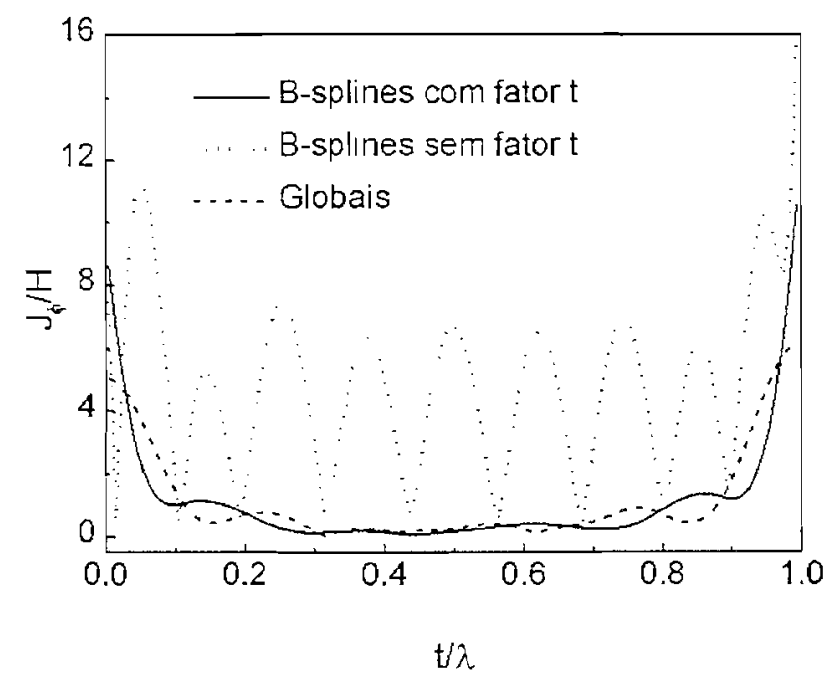

Figura 12. Componente $\oplus$ da densidade de corrente induzida sobre um cilindro condutor aberto por uma onda plana axialmente incidente.

para a representação por B-splines é potencialmente capaz de estabilizar a solução, ao mesmo tempo que simplifica as expressões resultantes devido a operadore diferencias no núcleo da equação integral em (1).

Duas observações adicionais podem ser feitas com respeito à implementação de B-splines como funções base. Em primeiro lugar, tais funções são facilmente adaptáveis à utilização de técnicas de AMG (adaptative mesh grading), ao se redistribuir a posição dos knots. A motivação principal é a possibilidade de concentrar maior número de knots em regiões críticas (de alta curvatura ou descontinuidades geométricas) onde se esperam componentes espectrais de mais alta frequiência. Isto poderia ser feito, por exemplo, através de uma

parametrização dada pela média ponderada do comprimento de arco, curvatura local e proximidade de bordas. Em segundo lugar, B-splines também estão presentes no contexto da Análise de Multiresolução (MRA). Utilizando B-splines como ponto de partida (funções de escala) uma sequiência de sub-espaços wavelet podem ser gerados [17], com convergência assintótica a funções de Gabor [15] (também otimamente concentradas no espaço de fase). No caso de um objeto espalhador complexo, incorporando tanto regiões de geometria suave como regiões de alta descontinuidade geométrica, a complementação da expansão por B-splines com wavelets nas regiōes críticas é potencialmente atrativa, pelo fato de funções wavelet 
minimizarem a interação mútua, o que por sua vez torna esparsa a região correspondente da matriz impedância.

\section{APÊNDICE A - EXPRESSÕES PARA OS ELEMENTOS DA MATRIZ IMPEDÂNCIA}

A seguir apresenta-se as integrais que definem os elementos da matriz impedância em (6):

$$
\begin{aligned}
& \left(Z_{m}^{u}\right)_{i j}=\int_{0}^{T} d t^{\prime} \int_{0}^{T} d t\left(\Gamma_{m}^{u} t_{i}^{\prime}(t) b_{j}^{i}\left(t^{\prime}\right)+\frac{1}{j \omega \varepsilon} G_{m}^{(j)} \frac{\partial t_{i}^{\prime}(t)}{\partial t} \frac{\partial b_{j}^{t}\left(t^{\prime}\right)}{\partial t^{\prime}}\right) \\
& \left(Z_{m}^{t \phi}\right)_{i j}=\int_{0}^{T} d t^{\prime} \int_{0}^{T} d t\left(\rho T_{m}^{\phi t} t_{i}^{t}(t) b_{j}^{\rho}\left(t^{\prime}\right)+\frac{m}{\omega \varepsilon} G_{m}^{(3)} \frac{\partial t_{i}^{i}(t)}{\partial t} b_{j}^{\circ}\left(t^{\prime}\right)\right) \\
& \left(Z_{m}^{\phi}\right)_{i j}=\int_{0}^{T} d t^{\prime} \int_{0}^{T} d t\left(\rho \Gamma_{m}^{t \phi} t_{i}^{\phi}(t) b b_{j}^{t}\left(t^{\prime}\right)-\frac{m}{\omega \varepsilon} G_{m}^{(3)} t_{i}^{\phi}(t) \frac{\partial b_{j}^{t}\left(t^{\prime}\right)}{\partial t^{\prime}}\right) \\
& \left(Z_{\mathrm{m}}^{\phi \phi}\right)_{\mathrm{ij}}=\int_{0}^{\mathrm{T}} \mathrm{dt} \mathrm{t}^{\mathrm{T}} \int_{0}^{\mathrm{d} t}\left(\rho \rho^{\prime} \Gamma_{\mathrm{m}}^{\phi \phi} \mathrm{t}_{\mathrm{i}}^{\phi}(\mathrm{t}) \mathrm{b}_{\mathrm{j}}^{\phi}\left(\mathrm{t}^{\prime}\right)+\frac{\mathrm{m}^{2}}{\omega \varepsilon} \mathrm{G}_{\mathrm{m}}^{(3)} \mathrm{t}_{\mathrm{i}}^{\phi}(\mathrm{t}) \mathrm{b}_{\mathrm{j}}^{\phi}\left(\mathrm{t}^{\prime}\right)\right)
\end{aligned}
$$

com

$$
\begin{aligned}
& \rho^{\prime}=\rho\left(t^{\prime}\right) \\
& \Gamma_{m}^{t t}=j \omega \mu\left(\operatorname{sen} v \operatorname{sen} v^{\prime} G_{m}^{(1)}+\cos v \cos v^{\prime} G_{m}^{(3)}\right) \\
& \Gamma_{m}^{t \phi}=-\omega \varepsilon \operatorname{sen} \nu G_{m}^{(2)} \\
& \Gamma_{m}^{\phi t}=\omega \varepsilon \operatorname{sen} \nu^{\prime} G_{m}^{(2)} \\
& \Gamma_{m}^{\phi \phi}=j \omega \mu G_{m}^{(1)} \\
& G_{m}^{(1)}=\int_{0}^{\pi} d \delta \cos \delta \cos (m \delta) G(R) \\
& G_{m}^{(2)}=\int_{0}^{\pi} d \delta \cos \delta \operatorname{sen}(m \delta) G(R) \\
& G_{m}^{(2)}=\int_{0}^{\pi} d \delta \cos (m \delta) G(R) \\
& G(R)=\exp (-j k R) / R \\
& R \equiv\left|\vec{r}^{\prime}-\vec{r}\right|=\sqrt{r^{\prime 2}+r^{2}-2 r r^{\prime} \cos \delta}
\end{aligned}
$$

$v$ é o ângulo formado entre a direção do unitário $\hat{t}$ e o eixo $z$, sendo positivo se $\hat{t}$ aponta para fora do eixo e negativo se aponta contra o eixo. $b_{i}^{p}(t)$ representam as funções base em (4) e $t_{i}^{p}(t)$ denotam as funções teste. No método de Galerkin (alternativa aqui adotada) estas funções são iguais. As integrais acima são calculadas numericamente. Detalhes sobre os procedimentos de cálculo podem ser obtidos em [25] Apenas metade dos elementos precisam ser calculado devido às relações de simetria:

$$
\begin{aligned}
& \left(Z_{-\mathrm{m}}^{\mathrm{tt}}\right)_{i j}=\left(Z_{\mathrm{m}}^{\mathrm{tt}}\right)_{i j} ; \\
& \left(Z_{-\mathrm{m}}^{\mathrm{t} \phi}\right)_{\mathrm{ij}}=-\left(Z_{\mathrm{m}}^{\mathrm{t} \phi}\right)_{\mathrm{ij}} ; \\
& \left(Z_{-\mathrm{m}}^{\phi \mathrm{t}}\right)_{\mathrm{ij}}=-\left(Z_{\mathrm{m}}^{\mathrm{t}}\right)_{\mathrm{ij}} ; \\
& \left(Z_{-\mathrm{m}}^{\phi \phi}\right)_{\mathrm{ij}}=\left(Z_{\mathrm{m}}^{\phi \phi}\right)_{i j}
\end{aligned}
$$

As integrais em (A1)-(A4) apresentam singularidades quando os pontos de fonte e observação coincidem, i. e., quando $\mathrm{R}=0$. Nestes pontos deve ser efetuada uma extração das singularidades antes da integração numérica (apêndice C).

\section{APÊNDICE B - VETORES EXCITAÇÃO}

Para o caso de o campo elétrico incidente ser uma onda plana linearmente polarizada (LP) na direção $\overrightarrow{\mathrm{p}}$ e propagando-se na direção $\overrightarrow{\mathrm{k}}_{\mathrm{t}}$, tem-se:

$$
\overrightarrow{\mathbf{E}}_{\text {inc }}=\exp \left(-j \overline{\mathbf{k}}_{t} \cdot \overline{\mathbf{r}} \overline{\mathbf{p}}\right.
$$

com

$\vec{k}_{t}=\frac{2 \pi}{\lambda}\left(\hat{x} \operatorname{sen} \theta_{t} \cos \phi_{t}+\hat{y} \operatorname{sen} \theta_{t} \operatorname{sen} \phi_{t}+\hat{z} \cos \theta_{i}\right)$

$\left(\theta_{\mathrm{t}}, \dot{\phi}_{\mathrm{t}}\right)$ define a direção de incidência e vetores unitários ortogonais à $\overrightarrow{\mathrm{k}}_{\mathrm{t}}$. Qualquer polarização $\overrightarrow{\mathrm{p}}$ pode ser decomposta na direção definida por $\theta_{\mathrm{t}}$ e na direção definida por $\phi_{\mathrm{t}}$. No caso de campo incidente LP na direção $\phi_{\mathrm{t}}$, o vetor excitação definido em (7) será dado por:

$$
\begin{aligned}
& \left(V_{m}^{c}\right)_{i}=-j^{m} \pi \exp \left(-j m \phi_{t}\right) \int_{0}^{T} t_{i}^{t}(t) \operatorname{sen} v\left(J_{m+1}(\zeta)+J_{m-1}(\zeta)\right) \exp (-j \gamma) d t \\
& \left(V_{m}^{\varphi}\right)_{i}=j^{m+1} \pi \exp \left(-j m \phi_{t}\right) \int_{0}^{T} \rho t_{i}^{\phi}(t)\left(J_{m+1}(\zeta)-J_{m-1}(\zeta)\right) \exp (-j \gamma) d t
\end{aligned}
$$

sendo $\zeta=k \rho \operatorname{sen} \theta_{\mathrm{t}}$ e $\gamma=k z \cos \theta_{\mathrm{t}} . \mathrm{J}_{\mathrm{m}}(\zeta)$ é a função de Bessel (primeira espécie) de ordem $\mathrm{m}$. No caso de campo incidente LP na direção $\theta_{t}$ a excitação é escrita como:

$\left(V_{m}^{\prime}\right)_{i}=j^{m} \pi \exp \left(-j m \phi_{1}\right)$.

$$
\begin{gathered}
\int_{0}^{T} \mathrm{t}_{\mathrm{i}}^{\mathrm{t}}(\mathrm{t})\left(\mathrm{j \operatorname {sen }} v \cos \theta_{t}\left(\mathrm{~J}_{\mathrm{m}-1}(\zeta)-\mathrm{J}_{\mathrm{m}-1}(\zeta)\right)-2 \cos v \operatorname{sen} \theta_{\mathrm{r}} \mathrm{J}_{\mathrm{m}}(\zeta)\right) \exp (-\mathrm{j} \gamma) \mathrm{dt} \\
\left.\left(V_{m}^{\phi}\right)_{i}=j^{m} \pi \exp -j m \phi_{t}\right) \int_{0}^{T} \mu_{i}^{\phi}(t) \cos \theta_{t}\left(J_{m+1}(\zeta)+J_{m-1}(\zeta)\right) \exp (j \gamma) d t
\end{gathered}
$$

Para incidencia axial, apenas os modos $m= \pm 1$ serão excitados.

Um modelo de campo radiado bastante útil na representação de alimentadores para antenas reletoras é o de 
uma onda esférica com distribuição de potência definida em dois planos como função do ângulo de elevação:

$$
\vec{E}_{i n c}=\left\{\begin{array}{l}
\left(\hat{\theta} \cos \phi F_{E}(\theta)-\hat{\phi} \operatorname{sen} \phi F_{H}(\theta)\right) \exp (-j k R) / R ; \quad 0 \leq \theta \leq \pi / 2 \\
0 ; \quad \theta>\pi / 2
\end{array}\right.
$$

onde $F_{E}$ e $F_{H}$ representam o diagrama do alimentador nos planos $\phi=0$ e $\phi=\pi / 2$ respectivamente. Esta fonte irradia na direção $+z$ e está localizada na origem do sistema de coordenadas. Nesta situação, os vetores excitação são dados por (somente modos $m= \pm 1$ são excitados):

$$
\begin{aligned}
& \left(V_{m}^{t}\right)_{i}=\pi \int_{0}^{T} t_{i}^{t}(t) F_{E}(\theta)\left(\cos \theta_{t} \operatorname{sen} v-\operatorname{sen} \theta_{t} \cos v\right) \frac{\exp (-j k R)}{R} d t \\
& \left(V_{m}^{\phi}\right)_{i}=j m \pi \int_{0}^{T} \rho t_{i}^{\phi}(t) F_{H}(\theta) \frac{\exp (-j k R)}{R} d t
\end{aligned}
$$

No caso de expansões mais gerais de ondas esféricas que incluem o comportamento em campo próximo pode ser necessário a adição à (A11) de termos comespondentes ao campo radial em (A10), dado por:

$$
\left.\overrightarrow{\mathrm{E}}_{\mathrm{inc}}\right|_{\mathrm{r}}=\hat{\mathrm{r}} \cos \phi \mathrm{F}_{\mathrm{R}}(\theta) \exp (-\mathrm{jkR}) / \mathrm{R}
$$

A integral (A11) possuirá então o termo adicional dado por:

$$
\left(V_{m}^{t}\right)_{i}=\pi \int_{0}^{T} t_{i}^{t}(t) F_{R}(\theta)\left(\operatorname{sen} \theta_{t} \operatorname{sen} v+\cos \theta_{t} \cos v\right) \frac{\exp (-j k R)}{R} d t(A 14)
$$

\section{APÊNDICE C -EXTRAÇÃO DAS SINGULA- RIDADES}

São dois os objetivos deste apêndice. O primeiro é rever o procedimento pseudo-analítico utilizado para a extração de singularidades nas integrais que definem os elementos da matriz impedância. O segundo é mostrar como esta extração pode ser feita de uma maneira mais apropriada utilizando-se $\mathrm{B}$-splines como funções base.

As integrais singulares que aparecem no cálculo dos elementos da matriz impedância têm a seguinte forma genérica:

$$
I=\int_{0}^{T} d t \int_{0}^{T} d t^{\prime} \alpha_{1}(t) \alpha_{2}\left(t^{\prime}\right) \int_{0}^{\pi} d \phi \cos \phi \cos n \phi \frac{e^{-j k R}}{R}
$$

sendo

$$
R=\left[\left(\rho-\rho^{\prime}\right)^{2}+\left(z-z^{\prime}\right)^{2}+4 \rho \rho^{\prime} \sin ^{2} \phi / 2\right]^{1 / 2}
$$

e $\alpha_{1}(t), \alpha_{2}(t)$ funções que dependem da escolha de funções base em particular. A integral acima possui uma singularidade em $\rho=\rho^{\prime}, z=z^{\prime}, \phi=0$ que não permite uma integração numérica adequada. Ela pode ser reescrita como:

$I=\int_{0}^{T} d t \int_{0}^{T} d t^{\prime} \alpha_{1}(t) \alpha_{2}\left(t^{\prime}\right)\left(\int_{0}^{\pi} d \phi\left(\cos \phi \cos n \phi \frac{e^{-j k R}}{R}-\frac{1}{R}\right)+\int_{0}^{\pi} \frac{d \phi}{R}\right)=I_{1}+I_{2}$

Desta forma, $\mathrm{I}_{1}$ é uma integral própria e a singularidade é isolada em $I_{2}$. Definindo: $R_{1}=\left[\left(\rho-\rho^{\prime}\right)^{2}+\left(z-z^{\prime}\right)^{2}\right]^{1 / 2}, \beta_{1}=\frac{2 \sqrt{\rho \rho^{\prime}}}{R_{1}} \quad e$ $\xi=\phi / 2$; então:

$I_{2}=2 \int_{0}^{T} d t \int_{0}^{T} d t^{\prime} \alpha_{1}(t) \alpha_{2}\left(t^{\prime}\right) \int_{0}^{\pi / 2} \frac{d \xi}{R_{1} \sqrt{\left(1+\beta_{1}^{2} \sin ^{2} \xi\right)}}$
$=2 \int_{0}^{T} d t \int_{0}^{T} d t^{\prime} \alpha_{1}(t) \alpha_{2}\left(t^{\prime}\right) \frac{K\left(\beta_{2}\right)}{R_{2}}$

$\operatorname{com} R_{2}=\left[\left(\rho+\rho^{\prime}\right)^{2}+\left(z-z^{\prime}\right)^{2}\right]^{/ 2} ; \beta_{2}=\frac{2 \sqrt{\rho \rho^{\prime}}}{R_{2}}$

$\mathrm{K}\left(\beta_{2}\right)$ é a integral elíptica completa do primeiro tipo. A integral em $\phi$ já esta resolvida mas a integral acima ainda é singular quando $\rho=\rho^{\prime}$ e $z=z^{\prime}$ (ou, equivalentemente, $\left.t=t^{\prime}\right)$. O comportamento de $K\left(\beta_{2}\right)$ para $t \rightarrow t^{\prime}$ é dado por:

$\lim _{t \rightarrow t^{\prime}} \frac{K\left(\beta_{2}\right)}{R_{2}}=\frac{1}{2 \rho}\left[\ln 4+\ln R_{2}-\ln R_{1}\right]$

Apenas o último termo acima é singular. Adicionando-o e subtraindo-o de $\mathrm{I}_{2}$, pode-se escrever:

$I_{2}=I_{21}-I_{22}=2 \int_{0}^{T} d t_{0}^{T} d t^{\prime} \alpha_{1}(t) \alpha_{2}\left(t^{\prime}\right)\left[\frac{K\left(\beta_{2}\right)}{R_{2}} \div \frac{\ell n R_{1}}{2 \rho}\right]-\int_{0}^{T} d t_{0}^{T} d t^{\prime} \alpha_{1}(t) \alpha_{2}\left(t^{\prime}\right) \frac{\ell n R_{1}}{\rho}$

$I_{21}$ é uma integral própria e pode ser calculada numericamente. $I_{22}$ é re-escrita como: $I_{22}=\frac{1}{2} \int_{0}^{T} d t \frac{\alpha_{1}(t)}{\rho} \sum_{i=1}^{N} \int_{\bar{\varepsilon}_{i}} d t^{\prime} \alpha_{2}\left(t^{\prime}\right) \ln R_{1, i}^{2}$

onde $o$ intervalo $[0, \mathrm{~T}]$ foi subdividido em $\mathrm{N}$ sub-intervalos. Cada sub-intervalo (se suficientemente reduzido) define um segmento da curva $C$ onde a dependência de $\rho^{\prime}$ e $z^{\prime}$ com $\mathrm{t}^{\prime}$ pode ser linearizada: $\rho^{\prime}=\rho_{i}^{\prime}+a_{i}\left(t^{\prime}-t_{i}^{\prime}\right)$;

$$
z^{\prime}=z_{i}^{\prime}+b_{i}\left(t^{\prime}-t_{i}^{\prime}\right) \text { e } R_{1, i}^{2}=\left(t^{\prime}-t_{0 i}\right)^{2}+t_{1 i}^{2}
$$

$\operatorname{com} t_{0 i}=t_{0 i}\left(t_{i}^{\prime}, z_{i}^{\prime}, \rho_{i}^{\prime}, a_{i}, b_{i}\right)$ e $t_{1 i}=t_{1 i}\left(z_{i}^{\prime}, \rho_{i}^{\prime}, a_{i}, b_{i}\right)$.

Em cada segmento $\varepsilon_{\mathrm{i}}$ a função $\alpha_{2}\left(t^{\prime}\right)$ é proporcional a função base $b_{i}^{p}\left(t^{\prime}\right)$. As integrais em cada segmento $\varepsilon_{i}$ podem ser calculadas analiticamente utilizando-se uma aproximação local na forma: 
$\alpha_{2}\left(t^{\prime}\right) \approx a_{0}+a_{1} t^{\prime}+a_{2} t^{\prime 2}+a_{3} t^{\prime 3}$ pois as integrais do tipo

$$
I_{22 i}=\int t^{n} \ln \left[\left(t-t_{0}\right)^{2}+t_{1}^{2}\right] d t \quad \mathrm{n}=0,1,2,3 .
$$

possuem solução analítica. Com o uso de B-splines como funções base, a aproximação local acima é exata pois possui localmente a mesma forma polinomial que as B-splines (os coeficientes são exatos), destarte não introduzindo erros adicionais na aproximação.

\section{REFERÊNCIAS}

[1] R. F. Harrington, "The Method of Moments in Electromagnetics", J. Electromag. Waves and Appl., vol.1, no.3, pp.181-200, 1987.

[2] J. R. Mautz e R. F. Harrington, "Radiation and Scattering from Bodies of Revolution" , Appl. Sci. Res., vol.20, pp.405-435, 1969.

[3] A. W. Glisson e D. R. Wilton, "Simple and Efficient Numerical Methods for Problems of Electromagnetic Radiation and Scattering from Surfaces", IEEE Trans. Antennas Propag., vol.28, no.5, pp.593-603, 1980.

[4] L. N. Medgyesi-Mitschang e C. Eftimiu, "Scattering from Wires and Open Circular Cylinders of Finite Length Using Entire Domain Galerkin Expansions", IEEE Trans. Antennas Propag., vol.30, no.4, pp.628636, 1982.

[5] M. R. Barclay e W. V. T. Rusch, "Moment-Method Analysis of Large, Axially Symmetric Reflector Antennas Using Entire-Domain Functions", IEEE Trans. Antennas Propag., vol.39, no.4, pp.491-496, 1991.

[6] E. Alanen, "Pyramidal and Entire Domain Basis Functions in the Method of Moments", J. Electromag. Waves and Appl., vol.5, no.3, pp.315-329, 1991.

[7] G. F. Herrmann, "Note on Interpolational Basis Functions in the Method of Moments", IEEE Trans. Antennas Propag., vol.38, no.1, pp.134-137, 1990.

[8] O. M. Bucci, G. D'Elia, R. Pierri, 'Exact Sampling Approach for Reflector Antenna Analysis", IEEE Trans. Antennas Propag., vol.32, pp.1259-1262, 1984.

[9] G. F. Herrmann, "Application of Outgoing-Wave and Related Bandlimited Basis Functions", IEEE Antennas Propag. Int. Symp. 1990 Digest, pp.1384-1387, 1990.

[10] F. L. Teixeira, e J. R. Bergmann, "Global vs. Bandlimited Basis Functions in the Analysis of Axisymmetric Reflector Antennas", IEEE Antennas Propag. Int. Symp. 1995 Digest, pp.1166-1169, 1995.
[11] F. L. Teixeira, e J. R. Bergmann, "Spatial-Frequency Localized Representations for Integral Equation Reflector Analysis", IEEE Antennas Propag. Int. Symp. 1996 Digest, 1996

[12] J. R. Bergmann, F. J. V. Hasselmann, L. P. N. Costa, F. L. Teixeira e C. G. do Rego, "On the Interpolation of Numerically Defined Surfaces in Collimated-Beam Dual Reflector Antennas", Microwave and Optical Tech. Lett., vol. 7, no.4, pp.209-212; 1994.

[13] J. R. Bergmann, e J. C. Costa, "Dual Offset Antennas with Spherical Main Reflector for Small Earth Station", IEEE Trans. Antennas Propag., vol. 41, no.4, pp.355-356, 1993.

[14] D. C. Jenn. "Application of Integral Equation Theory to Reflector Antenna Analysis", Ph.D. dissertation, University of Southern California, Los Angeles, 1987.

[15] M. Unser, A. Aldroubi, M. Eden, "On Asymptotic Convergence of B-Spline Wavelets to Gabor Functions", IEEE Trans. Inf. Theory, vol. 38, pp.864872,1992

[16] C. de Boor, "On Calculating with B-Splines", J. Approx. Theory, vol. 6, pp.50-62, 1972

[17] Goswami, A. K. Chan and C. K. Chui, "On Solving First-Kind Integral Equations Using Wavelets on a Bounded Interval", IEEE Trans. Antennas Propag., vol.43, no.6, pp.614-622, 1995

[18] W. A.Davis e R. Mittra, "A New Approach to the Thin Scatterer Problem Using the Hybrid Equations", IEEE Trans. Antennas Propag., vol.25, no.3, pp.402-406, 1977

[19] F. L. Teixeira, F. J. S. Moreira, J. R. Bergmann, "Shaped Reflector Antennas: An Efficient Approach for the Design Procedure", ", IEEE Antennas Propag. Int. Symp. 1993 Digest, pp.266-269, 1993

[20] D. -W. Huan, Y. Rahmat-Samii, "A Generalized Diffraction Synthesis Tecnique for high Performance Reflector Antennas", IEEE Trans. Antennas Propag., vol.43, pp.27-40, 1995

[21] J. R. Bergmann, F. J. V. Hasselmann, F. L. Teixeira, C. G. Rego, "A Comparison Between Techniques for Global Surface Interpolation in Shaped Reflector Analysis", IEEE Trans. Antennas Propag., vol.42, pp.47-53, 1994

[22] G. Fraceschetti, "Reflector Antenna Analysis", SBMO 1985 Int. Symp. Dig., pp.67-74, 1985.

[23] J. Van Bladel, Singular Eletromagnetic Fields and Sources, Oxford University Press, 1991.

[24] D. R. Wilton and S. Govind, "Incorporation of Edge Conditions in Moment Method Solutions", IEEE 
Trans. Antennas Propag., vol.25, no.6, pp.845-850, 1977.

[25] L. Teixeira, "Aplicação do Método dos Momentos para a Anélise de Refletores Circularmente Simétricos", Tese de Mestrado, Departamento de Engenharia Elétrica -PUC/RJ, março de 1995.

José Ricardo Bergmann nasceu em Porto Alegre em 25 de março de 1953. Graduou-se em Engenharia Elétrica pela Universidade Federal do Rio Grande do Sul em dezembro de 1975. Recebeu o título de Mestre em Engenharia Elétrica (Msc) pelo Instituto Militar de Engenharia em fevereiro de 1979 e o título de Doutor em Engenharia Elétrica (PhD) pela University of London em dezembro de 1986. É professor associado do Centro de Estudos em Telecomunicações e coordenador de pós-graduação do Departamento de Engenharia Elétrica da Pontifícia Universidade Católica do Rio de Janeiro, estando vinculado a esta universidade desde 1979. Seus principais interesses em pesquisa são eletromagnetismo aplicado, síntese e análise de antenas refletoras e antenas para comunicaçōes via satélite. É atualmente chairman do Capítulo Conjunto Rio de Janeiro das Sociedades AP/MTT/ED do IEEE.

Fernando Lisboa Teixeira: biografia não disponível. 